Open Access

\title{
Assessment of an automated capillary system for Plasmodium vivax microsatellite genotyping
}

\author{
Paulo Manrique ${ }^{1}$, Mari Hoshi ${ }^{1}$, Manuel Fasabi ${ }^{4}$, Oscar Nolasco ${ }^{1}$, Pablo Yori ${ }^{3}$, Martiza Calderón², \\ Robert H. Gilman ${ }^{3}$, Margaret N. Kosek ${ }^{3}$, Joseph M. Vinetz ${ }^{1,2^{*}}$ and Dionicia Gamboa ${ }^{1,5^{*}}$
}

\begin{abstract}
Background: Several platforms have been used to generate the primary data for microsatellite analysis of malaria parasite genotypes. Each has relative advantages but share a limitation of being time- and cost-intensive. A commercially available automated capillary gel cartridge system was assessed in the microsatellite analysis of Plasmodium vivax diversity in the Peruvian Amazon.

Methods: The reproducibility and accuracy of a commercially-available automated capillary system, QIAxcel, was assessed using a sequenced PCR product of 227 base pairs. This product was measured 42 times, then 27 P. vivax samples from Peruvian Amazon subjects were analyzed with this instrument using five informative microsatellites. Results from the QIAxcel system were compared with a Sanger-type sequencing machine, the ABI PRISM ${ }^{\circledR} 3100$ Genetic Analyzer.

Results: Significant differences were seen between the sequenced amplicons and the results from the QIAXcel instrument. Different runs, plates and cartridges yielded significantly different results. Additionally, allele size decreased with each run by 0.045 , or 1 bp, every three plates. QIAxcel and ABI PRISM systems differed in giving different values than those obtained by ABI PRISM, and too many (i.e. inaccurate) alleles per locus were also seen with the automated instrument.

Conclusions: While P. vivax diversity could generally be estimated using an automated capillary gel cartridge system, the data demonstrate that this system is not sufficiently precise for reliably identifying parasite strains via microsatellite analysis. This conclusion reached after systematic analysis was due both to inadequate precision and poor reproducibility in measuring PCR product size.
\end{abstract}

Keywords: Malaria, Molecular biology, Genotyping, Microsatellites, Molecular epidemiology, Technology

\section{Background}

Molecular markers are widely used in the study of malaria and other infectious diseases [1]. Such markers allow for the differentiation of new and recurrent infections, tracing spread of clones of a pathogenic microorganism and discovering new introductions of infection

\footnotetext{
*Correspondence: jvinetz@ucsd.edu; dionigamboa@yahoo.com

${ }^{2}$ Division of Infectious Diseases, Department of Medicine, University

of California San Diego, La Jolla, CA, USA

${ }^{5}$ Departamento de Ciencias Celulares y Moleculares, Universidad Peruana

Cayetano Heredia, Lima, Peru

Full list of author information is available at the end of the article
}

on scales both large and small [2-5]. Molecular markers allow for genetic diversity studies of pathogen populations, their substructure and relationships among those sub-populations. Such data underpin fine scale analysis of infectious disease transmission dynamics [6-13], and allow us to infer the disease history and pathogen migration patterns, of particular importance in current malaria control- and elimination-focused research [14-17].

Different methods and markers have been used to study Plasmodium genetic diversity and population structure. A key feature of such studies is that various marker types evolve at different rates, being driven by complex 
mechanisms [10]. With regard to Plasmodium vivax, markers have included PCR-restriction fragment length polymorphism (PCR-RFLP) using a variety of genes including PvCSP, PvMSP3- $\alpha$ [18-20] and PvMSP3- $\beta$ $[8,9,19-23]$. Another marker type is based on DNA sequence and single nucleotide polymorphisms (SNPs), which have been emphasized for global comparisons [15, $16,24]$. In recent years, parasite microsatellites markers have been used specifically to assess population diversity and structure [10-13, 25, 26], because large numbers of such markers are present in Plasmodium falciparum and $P$. vivax genomes [24, 27-29]. Microsatellites are presumed to be neutral if they are not physically linked to genes under selection [30-32], and have sufficiently high mutation rates to allow for the identification of epidemiologically relevant diversification events over relatively short periods of time [10].

While different platforms and techniques have been used to generate the raw data for microsatellite genotyping, they tend to be expensive. Costs include fluorophore-labelled primers and a capillary electrophoresis platform, typically a Sanger-type sequencing machine. In this study, the precision and reproducibility of the QIAxcel Advanced System from Qiagen [33] was evaluated using a well-characterized, gold standard sequenced PCR product followed by analysis of $271 P$. vivax-infected blood samples from subjects living in the Peruvian Amazon. This platform was chosen based on reported performance characteristics primarily because fluorophore-labelled primers were not needed (hence the potential for cost saving). The main conclusion of this work, based on exhaustive analysis, should be a cautionary message for resource-limited settings, where reliance on hardware must be presaged by proper performance validation prior to investment of resources.

\section{Methods}

\section{Ethical considerations}

This study was approved by the Human Subjects Protection Program of the University of California, San Diego and the Comité de Ética of the Universidad Peruana Cayetano Heredia. Subjects provided written informed consent to participate in the study.

\section{Plasmodium vivax samples}

Samples for this study were obtained from three rural villages in the Peruvian Amazon region of Iquitos, Peru, in the Loreto Department, between 2005 and 2008: Santo Tomás (latitude -3.8094 , longitude -73.3419 ,), San José de Lupuna (latitude -3.74611 , longitude -73.3256) and Padrecocha (latitude -3.6997 , longitude -73.2786 ). Rural communities are located 9.8, 9.9 and $21.1 \mathrm{~km}$ from Iquitos, respectively (main urban area in the region); San
José de Lupuna and Padrecocha are accessible only by boat (Fig. 1).

Malaria diagnosis was carried out by microscopy using standard methods; for this reason only individuals with patent $P$. vivax parasitaemia were included in the study. Blood was obtained by venipuncture into EDTA tubes. Blood samples were frozen at $-20{ }^{\circ} \mathrm{C}$ and shipped on dry ice to Universidad Peruana Cayetano Heredia, Lima, Peru for laboratory analysis. All individuals that tested positive were treated with primaquine (1 week, $15 \mathrm{mg} /$ $\mathrm{kg}$ daily plus chloroquine) according to policies of the National Malaria Control Programme, Peruvian Ministry of Health.

\section{Accuracy, repeatability and reproducibility}

One pGEM T-Easy plasmid containing a region of gene Pf 18 S rRNA was amplified by PCR using forward primer PL1473F18 [5'-TAA CgA ACg AgA TCT TAA-3'] and reverse primer PL1679R18 [5'-gTT CCT CTA AgA AgC TTT-3'] [34]. This PCR product was sequenced on the ABI PRISM 3100 Genetic Analyzer, then run 42 times in 42 different plates among four different cartridges on the QIAxcel Advanced System machine. In this way data obtained from sequencing and from the QIAxcel Advanced System machine were compared to assess reproducibility. Statistical analysis was done using STATA 12 (College Station, TX, USA). Student's T test was used to test differences among outputs from different runs, plates and cartridges used on the QIAxcel platform, and to test differences between the QIAxcel with the reference gold standard method ABI PRISM 3100 Genetic Analyzer. To measure the effect of cartridge use on the outputs, a generalized linear mixed regression analysis was made [35]. To test whether the variance of the results from the QIAxcel machine was greater than expected compared to the ABI PRISM System, a one sample test of variance was used [36], assuming a maximum value of the standard deviation of the ABI PRISM system of 0.5 base pairs; additionally, the test was also run assuming a maximum standard deviation of one base pair. Finally, the accuracy of this QIAxcel platform was measured using five different microsatellite targets on 27 P. vivax samples. Three microsatellites (MS4, MS9 and MS15) were non-degenerate or perfect, indicating a unique motif repeat. The other two microsatellites were imperfect with mixed motifs; combination between discontinuous motifs, that are trunked with non-repetitive regions, and compounds motifs, with two or more different motifs. Each PCR product was analyzed in both platforms the QIAxcel Advanced System machine and the ABI PRISM system, and three replicates of MS9 and MS15 were done. Results differences between platforms were tested using Student's T test in STATA 12. 


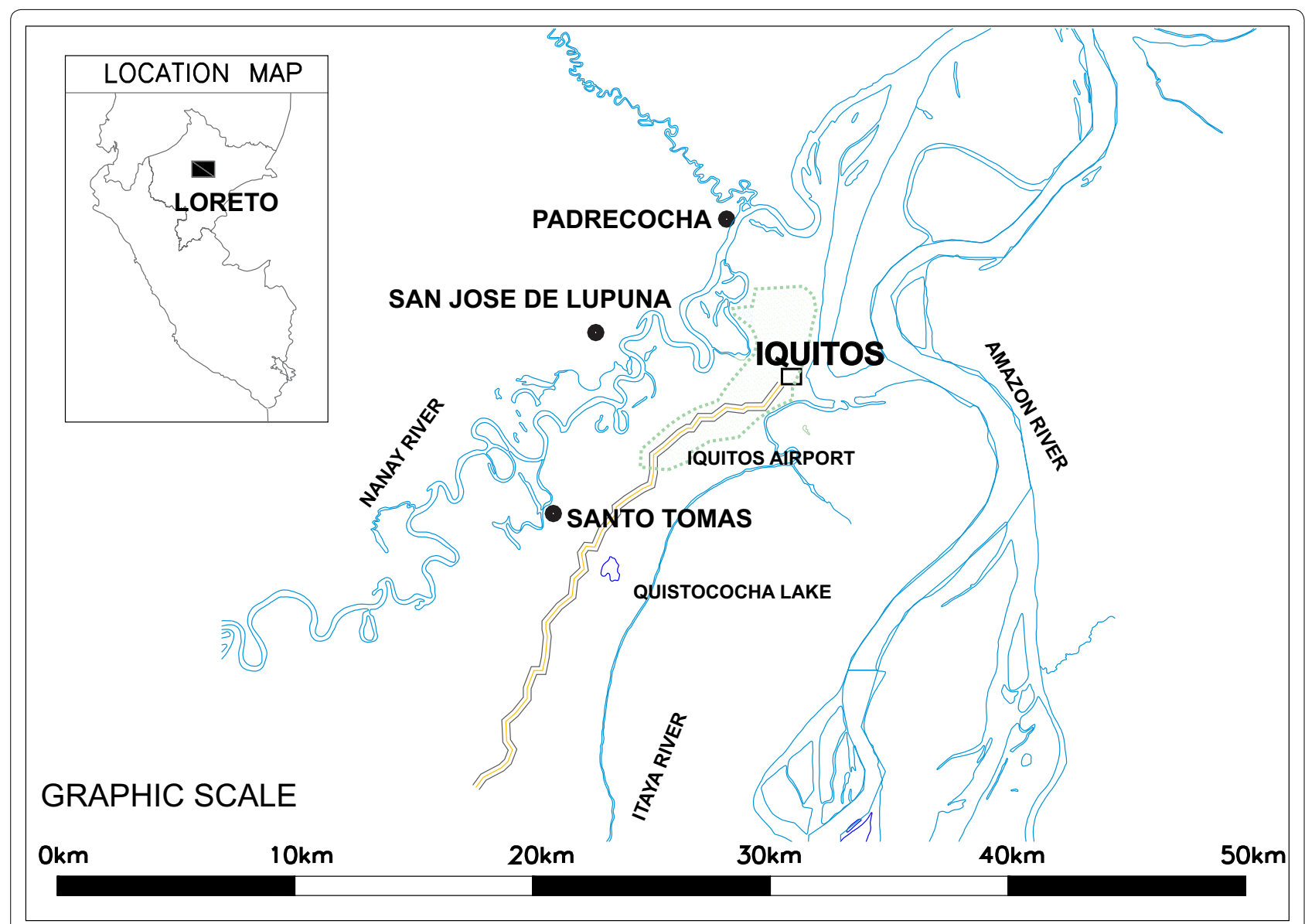

Fig. 1 Map of the study sites

\section{Microsatellite genotyping}

Genomic DNA was isolated from whole blood using the QIAamp DNA Blood Kit (QIAGEN, Valencia, CA, USA). Samples were genotyped using a set of 15 previously reported microsatellite markers [37, 38]. Five microsatellites were used to genotype 27 samples in a test for accuracy (MS4, MS6, MS9, MS15 and MS20), and all microsatellites were used to measure genetic diversity using $271 P$. vivax samples (primers are listed in Additional file 1). Five microliters of template DNA was used for PCR in a $50 \mu \mathrm{l}$ total volume for each individual reaction. The PCR product was then separated on a QIAxcel Advanced System (QIAGEN). The QIAxcel Advanced System from Qiagen is commercially presented as a novel means to replace gel analysis of DNA, RNA, and proteins. It consists of 12 independent capillaries placed in a cartridge. Each cartridge allows processing about 100 samples per capillary and 96 samples per PCR plate. This system is marketed to have a resolution of 3-5 bp. The PCR product sizes were estimated using the QIAxcel ScreenGel Software.

\section{Capillary electrophoresis analysis}

Electrophoresis runs on the QIAxcel Advanced System (QIAGEN) were performed according to manufacturer's recommendations. For each electrophoresis process, a QIAxcel DNA High Resolution Cartridge (Cat. no. 929002), a QX Alignment Marker 15 bp/600 bp (Cat. no. 929530), a standard QX DNA size marker $25-500$ pb v2.0 (Cat. no. 929560), and the OM 700 method, were used. Each cartridge was calibrated using QX Intensity Calibration Marker (cat. no. 929500) for its first use. Because five plates were processed per day (40 runs), the Separation buffer, the Wash buffer and the Alignment Marker on the buffer tray were renewed every day. The first well (position A1) of each plate was filled with $20 \mu \mathrm{l}$ of onetwentieth dilution of the standard QX DNA Size Marker 25-500 bp. The second well (position A2) was filled with Pf 18s rRNA PCR product as a reference control. The estimated size of this product was used to confirm or correct the size of the samples between plates. The third and fourth well was filled with the positive and negative PCR control samples, respectively. The positive control was a 
monoclonal sample with high parasitaemia used to confirm the effectiveness of the PCR. It was also used to see the presence of non-specific and stutters products. Additionally, this product also helped to correct the inferred size of the samples.

ABI PRISM system electrophoresis runs were carried out using a 4-capillary array of $36 \mathrm{~cm}$ in combination with 3130 POP-4TM Polymer. The GeneScan 500 LIZ dye was used as Size Standard, and primers were labelled with 6FAM, VIC, NED and PET dyes. Each plate well was filled with $0.25 \mu \mathrm{l}$ of GeneScan $500 \mathrm{Liz}, 9.25 \mu \mathrm{l}$ of $\mathrm{Hi}-\mathrm{Di}$ Formamide, and $1 \mu \mathrm{l}$ of one-tenth dilution of the PCR product. A monoclonal sample was used as positive control, and Human DNA as negative control.

\section{Microsatellite analysis}

The PCR product sizes were estimated using the QIAxcel ScreenGel Software and the GeneMapper v3.0 for QIAxcel and ABI PRISM results, respectively. Peaks with more than $50 \mathrm{RFU}$ (Relative fluorescence units) in the electropherogram were interpreted as alleles, and if additional peak of at least one-third the height of the predominant peak was seen it was also scored as allele. Finding more than one allele was interpreted as a polyclonal infection containing two or more genetically distinct clones in the same sample. Missing data (no amplifications) was reported by loci but were not considered here for defining haplotypes.

Microsatellite data were entered and cleaned using Microsoft Excel, then formatted using an Excel complement called GenAlEx 6.501 [39]. The expected heterozygosity $(\mathrm{He})$ parameter was estimated, defined as:

$$
\mathrm{H}_{\mathrm{e}}=[\mathrm{n} /(\mathrm{n}-1)]\left[1-\sum_{\mathrm{i}=1}^{\mathrm{L}} \mathrm{p}_{\mathrm{i}}^{2}\right],
$$

where $\mathrm{n}$ is the number of isolates analyzed and $\mathrm{pi}$ is the frequency of the ith allele $(i=1, \ldots, L)$ in the population [40].

Expected heterozygosity $(\mathrm{He})$ gives the average probability that a pair of alleles randomly selected from the population is different. Haplotypes, described as unique allelic combinations of the 15 loci analyzed, were determined in monoclonal samples using GenAlEx.

\section{Results}

Accuracy, repeatability and reproducibility of the QIAxcel Advanced Capillary System

A sequenced PCR product of 227 base pairs (Additional file 2) was run 42 times in four different cartridges (5, 13, 13, and 11 times per each cartridge) on the QIAxcel Advanced System, giving a mean value of 223.619 base pair $(S D=1.62229)$, and a range from 220 to $226 \mathrm{bp}$.
There were significant differences between the sequenced values and the results from the QIAxcel Advanced System (Student's t test, $p=0.0029$ ). Moreover, there were significant differences between different runs, plates and cartridges (Student's t test, $p=0.0275$ ) (Fig. 2). Additionally, 18 samples were run at least five times in the same run to test reproducibility among capillaries. Using the 'one sample test of variance' showed significant differences even at a maximum standard deviation value of $1(p=0.0003)$.

The effect of the time of use of each cartridge was explored by using a mixed linear regression analysis. A statistically significant decrease of -0.0449 base pairs from one run to the next ( $95 \% \mathrm{CI}-0.0533$ to -0.0365 ) was observed in the allele size each time a sample was evaluated; and a decrease of -0.3595 base pairs ( $95 \% \mathrm{CI}$ -0.4267 to -0.2922$)$ was found each time a plate was run. Furthermore, from the four cartridges used, the results given by the second cartridge were -2.657 base pairs $(95 \%$ $\mathrm{CI}-3.447$ to -1.868 ) lower than those given by the first one (Fig. 3). In the case of the third and fourth cartridges the results were $-1.042(95 \% \mathrm{CI}-1.832$ to -0.252$)$ and -1.387 (95\% CI -2.21 to -0.565 ) lower, respectively.

Comparing analysis of five microsatellites (MS4, MS6, MS9, M15 and MS20) from 27 samples, the automated QIAxcel advanced system showed higher size values than those obtained by the ABI PRISM system. On average, QIAxcel's size values were 7.022 base pairs higher (95\% CI 6.34-7.7). To test if differences between platforms were due to the label of primers, MS9 and MS15 were analyzed using both kind of primers in triplicates, and no significant differences were seen in MS9 products; however, significant differences were seen in MS15 products ( $\mathrm{p}$ value $<0.0001$ ). The numbers of alleles for MS4, MS6, MS9, MS15 and MS20 on the ABI PRISM system were $3,2,4,3$ and 4 , respectively. The corresponding

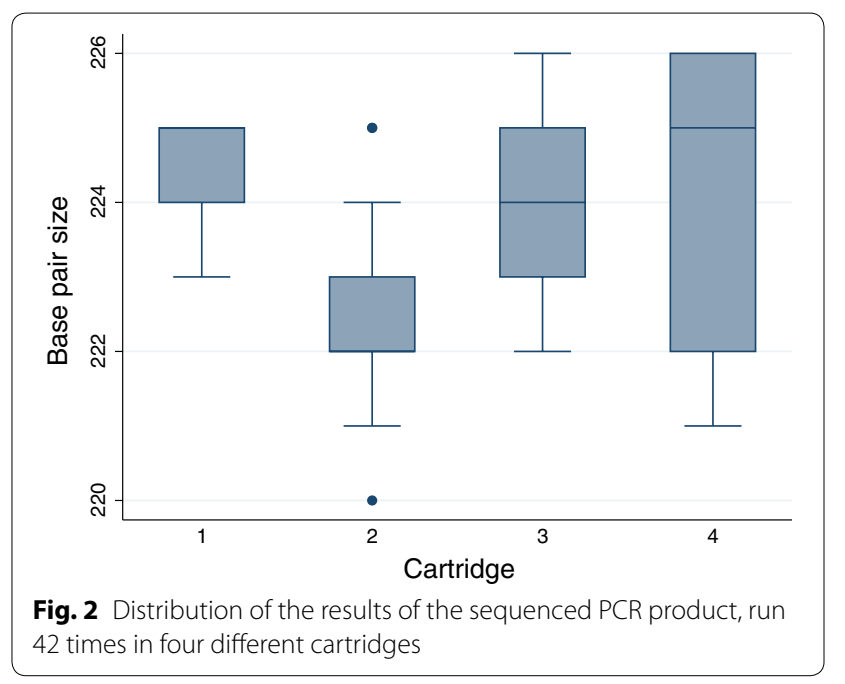




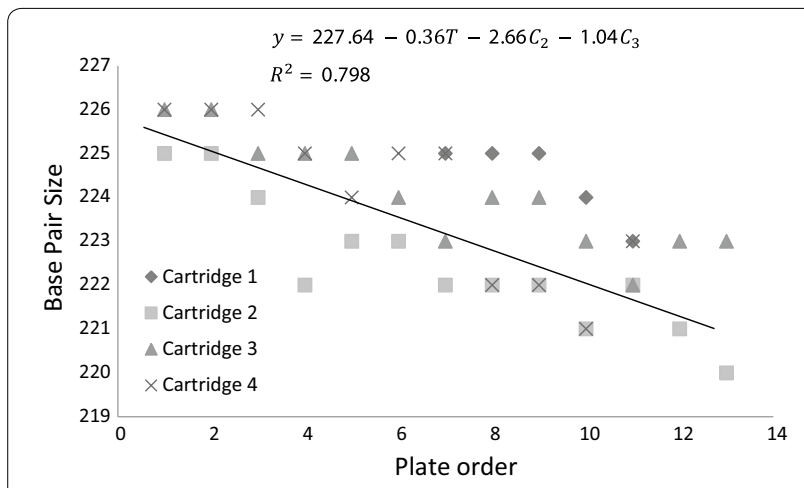

Fig. 3 Effect of the time of use on the outcome obtained from the QIAxcel Advanced System. Y-axis is the result of the QIAxcel (the results of the sequenced product) with four different cartridges, and the $X$-axis is the time order of each plate which was ran in the QIAXcel. $T$ is the time order of the plate in each cartridge and -0.36 is its effect on the outcome. C2, C3 and C4 are the second, third and fourth used cartridges and the values represent their effect on the outcome in respect to the first cartridge

allele numbers on the QIAxcel system were 7, 6, 5, 4 and 8 , respectively (summarized in Table 1 ; complete results in Additional file 3). Three of the 27 samples were polyclonal by ABI PRISM, but only two were detected as such by the QIAxcel system. Analysis of microsatellites MS6 and MS20 detected all polyclonal samples using the ABI PRISM system, while MS15 was not. The other two markers did not detect any polyclonal sample. On the QIAxcel system only microsatellite MS15 was able to detect the polyclonal samples.

\section{Analysis of Plasmodium vivax samples from the Peruvian Amazon}

A total of $271 P$. vivax single infection samples were analyzed using samples obtained from three rural communities around Iquitos (Padrecocha, San José de Lupuna and Santo Tomás) from the years 2005 and 2008. In Padrecocha, 50 blood samples were taken in the year 2005; 50 blood samples were available from 2006. In San José de Lupuna, 15 blood samples obtained in 2006; 35 blood samples were from 2007; 20 blood samples were from 2008. From Santo Tomás, 40 blood samples were obtained in 2006; 30 blood samples in 2007; and 31 blood samples in 2008 (Table 2).

\section{Estimation of Plasmodium vivax genetic diversity}

Two-hundred and seventy-one P. vivax samples were genotyped using the automated QIAxcel Advanced System machine. Of the $271 P$. vivax samples, 49 samples were polyclonal; 13 (15\%) polyclonal infections were found in Padrecocha, $14(25 \%)$ in San José de Lupuna and $22(28 \%)$ in Santo Tomás (Table 2). Moreover, of the 222 monoclonal samples, 141 amplified all loci, 53 showed one null allele, 22 showed two null alleles, five showed three null alleles, and just one sample did not amplify 14 loci out of the 15 loci analyzed (Table 3 ).

Because of the insufficient accuracy and reproducibility obtained using the QIAxcel Advanced System, the base pair size result was transformed into an allele profile and coded. In this way, all base pair size results were ranked by size and a frequency histogram was made per each locus. Base pair sizes with consecutive results (for example, 242, 243, 244, 245, $246 \mathrm{bp)}$ were grouped into one unique allele based on frequency (Fig. 4). There were some base pair size results that were in the middle of two alleles and hence could not be defined. Each base pair size result that was not defined was labelled as zero or a null allele. As a result, of the 222 monoclonal samples, 103 samples had at least one undefined allele and just 72 samples had a complete profile (Table 3). Most microsatellites except MS4, MS5, MS15, and PV6635 lacked at least $5 \%$ of possible data (MS16 presented the highest missing data value with $18.55 \%$ ).

Table 1 Comparison of results using QIAxcel vs. ABI PRISM methods

\begin{tabular}{|c|c|c|c|c|c|c|c|c|c|c|}
\hline \multirow[t]{2}{*}{ Allele } & \multicolumn{2}{|l|}{ MS4 } & \multicolumn{2}{|l|}{ MS6 } & \multicolumn{2}{|l|}{ MS9 } & \multicolumn{2}{|l|}{ MS15 } & \multicolumn{2}{|l|}{ MS20 } \\
\hline & $A B I$ & QIAxcel & $A B I$ & QIAxcel & $A B I$ & QIAxcel & $A B I$ & QIAxcel & $A B I$ & QIAxcel \\
\hline 1 & $187(0.19)$ & $190(0.08)$ & $210(0.63)$ & $219(0.39)$ & $147(0.19)$ & $156(0.19)$ & $243(0.69)$ & $247(0.24)$ & $200(0.1)$ & $209(0.04)$ \\
\hline 2 & $193(0.63)$ & $191(0.08)$ & $229(0.33)$ & $220(0.18)$ & $155(0.04)$ & $165(0.11)$ & $249(0.14)$ & $248(0.45)$ & $206(0.2)$ & $210(0.07)$ \\
\hline 3 & $200(0.19)$ & $192(0.04)$ & & $221(0.07)$ & $157(0.11)$ & $166(0.04)$ & $258(0.17)$ & $254(0.14)$ & $220(0.07)$ & $211(0.04)$ \\
\hline 4 & & $197(0.32)$ & & $233(0.04)$ & $163(0.67)$ & $172(0.60)$ & & $262(0.17)$ & $222(0.63)$ & $216(0.15)$ \\
\hline 5 & & $198(0.36)$ & & $237(0.14)$ & & $173(0.07)$ & & & & $218(0.04)$ \\
\hline 6 & & $203(0.08)$ & & $238(0.18)$ & & & & & & $221(0.04)$ \\
\hline 7 & & $204(0.04)$ & & & & & & & & $230(0.44)$ \\
\hline 8 & & & & & & & & & & $231(0.19)$ \\
\hline
\end{tabular}

The table shows the base pair size of each allele. In parentheses the frequencies of each allele are denoted 
Table 2 Number of monoclonal and polyclonal samples by study site

\begin{tabular}{lcccr}
\hline Locality & Year & Monoclonal & Polyclonal & Total \\
\hline Padrecocha & 2005 & $43(86 \%)$ & $7(14 \%)$ & 50 \\
& 2006 & $44(88 \%)$ & $6(12 \%)$ & 50 \\
& Total & $87(87 \%)$ & $13(13 \%)$ & 100 \\
San Jose de Lupuna & 2006 & $14(93 \%)$ & $1(7 \%)$ & 15 \\
& 2007 & $26(74 \%)$ & $9(26 \%)$ & 35 \\
& 2008 & $16(80 \%)$ & $4(20 \%)$ & 20 \\
Santo Tomas & Total & $56(80 \%)$ & $14(20 \%)$ & 70 \\
& 2006 & $30(75 \%)$ & $10(25 \%)$ & 40 \\
& 2007 & $26(87 \%)$ & $4(13 \%)$ & 30 \\
& 2008 & $23(74 \%)$ & $8(26 \%)$ & 31 \\
Total & Total & $79(78 \%)$ & $22(22 \%)$ & 101 \\
& & $222(82 \%)$ & $49(18 \%)$ & 271 \\
\hline
\end{tabular}

The He index of samples from each geographical source of sample was $0.744 \pm 0.039,0.734 \pm 0.056$ and $0.716 \pm 0.052$, for Padrecocha, San José de Lupuna and Santo Tomas, respectively; He for the whole population was $0.779 \pm 0.04$. Microsatellite MS7 showed the lowest diversity in the whole population $(\mathrm{He}=0.375)$ as well as in the three different locations (He for MS7 in Padrecocha, San José de Lupuna and Santo Tomás was 0.536, 0.170 , and 0.296 , respectively). MS5 showed low diversity $(\mathrm{He}=0.539,0.343$ and 0.399 , respectively); however, its diversity was higher among the whole population $(\mathrm{He}=0.621)$. Microsatellites MS3 and MS1 showed a similar patterns. Microsatellites MS16, MS9 and MS8 showed the highest genetic diversity among localities and in the whole population (average $\mathrm{He}=0.92,0.91$ and 0.902 , respectively). Moreover, many of the alleles from the different markers used were in low frequency (frequency lower than $5 \%$, referred as minor allele frequency or MAF, Table 4).

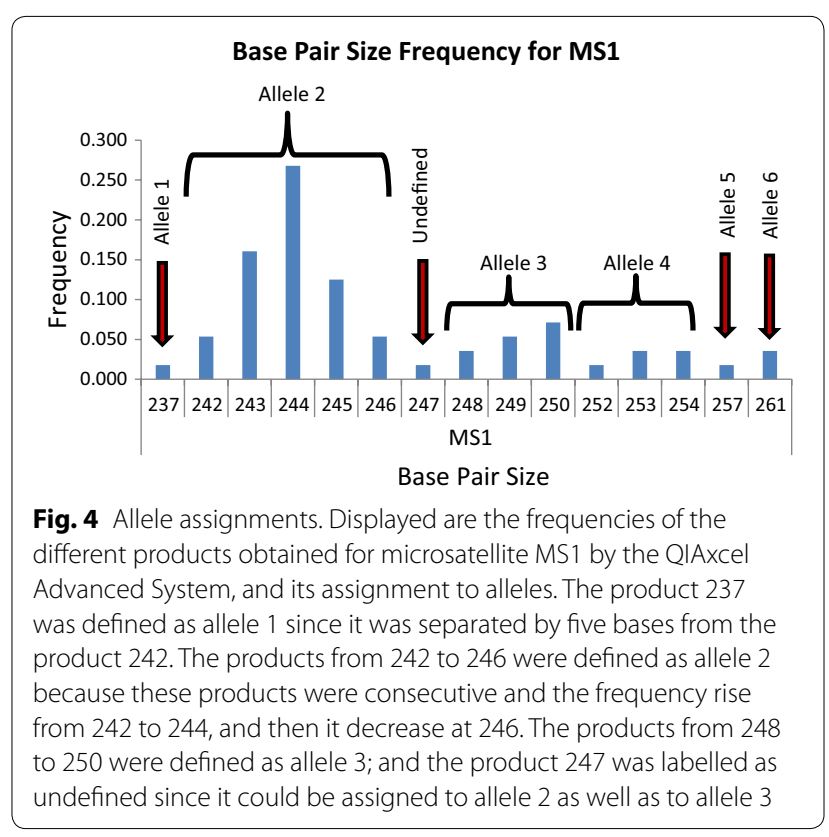

Among the 72 monoclonal infections with complete profiles, 62 haplotypes were found, of which 53 had unique haplotypes. None of the haplotypes were shared among the three populations, but one haplotype was shared in San José de Lupuna between the years of 2007 and 2008.

\section{Discussion}

This study aimed to explore the utility of a non-fluorophore-dependent, automated microsatellite analysis system that purportedly had two major advantages-higher throughput and lower cost-to obtain raw microsatellite marker data. However, the use of microsatellite data, like that of other markers [22], requires high precision and reproducibility. Hence, any platform used to generate

Table 3 Number of missing data in each study site

\begin{tabular}{|c|c|c|c|c|c|c|c|c|c|}
\hline \multirow{2}{*}{$\begin{array}{l}\text { Number } \\
\text { of missing } \\
\text { data }\end{array}$} & \multicolumn{2}{|l|}{ Padrecocha } & \multicolumn{2}{|c|}{ San Jose de Lupuna } & \multicolumn{2}{|l|}{ Santo Tomas } & \multicolumn{3}{|l|}{ Total } \\
\hline & Null alleles & $\begin{array}{l}\text { Undefined } \\
\text { alleles }\end{array}$ & Null alleles & $\begin{array}{l}\text { Undefined } \\
\text { alleles }\end{array}$ & Null alleles & $\begin{array}{l}\text { Undefined } \\
\text { alleles }\end{array}$ & Null alleles & $\begin{array}{l}\text { Undefined } \\
\text { alleles }\end{array}$ & $\begin{array}{l}\text { Missing data } \\
(\%)\end{array}$ \\
\hline 0 & 54 & 47 & 48 & 32 & 39 & 40 & 141 & 119 & $72(32.4)$ \\
\hline 1 & 23 & 22 & 6 & 18 & 24 & 29 & 53 & 69 & $72(32.4)$ \\
\hline 2 & 7 & 9 & 2 & 6 & 13 & 7 & 22 & 22 & $49(22.1)$ \\
\hline 3 & 3 & 6 & 0 & 0 & 2 & 1 & 5 & 7 & $16(7.2)$ \\
\hline 4 & 0 & 1 & 0 & 0 & 0 & 2 & 0 & 3 & $9(4.1)$ \\
\hline 5 & 0 & 2 & 0 & 0 & 0 & 0 & 0 & 2 & $3(1.4)$ \\
\hline 14 & 0 & 0 & 0 & 0 & 1 & 0 & 1 & 0 & $1(0.5)$ \\
\hline Total & 87 & 87 & 56 & 56 & 79 & 79 & 222 & 222 & $222(100)$ \\
\hline
\end{tabular}


Table 4 Number of alleles and genetic diversity by locus by site

\begin{tabular}{|c|c|c|c|c|c|c|c|c|c|c|c|c|}
\hline \multirow{2}{*}{$\begin{array}{l}\text { Pop } \\
\text { Locus }\end{array}$} & \multicolumn{4}{|c|}{ Padrecocha } & \multicolumn{4}{|c|}{ San Jose de Lupuna } & \multicolumn{4}{|c|}{ Santo Tomas } \\
\hline & $\mathrm{N}$ & $\mathrm{Na}$ & MAF (\%) & $\mathrm{He}$ & $\mathrm{N}$ & $\mathrm{Na}$ & MAF (\%) & $\mathrm{He}$ & $\mathbf{N}$ & $\mathrm{Na}$ & MAF (\%) & $\mathrm{He}$ \\
\hline MS1 & 70 & 5 & $2(40)$ & 0.659 & 55 & 6 & $4(67)$ & 0.52 & 63 & 6 & $4(67)$ & 0.467 \\
\hline MS2 & 77 & 7 & $4(57)$ & 0.651 & 56 & 8 & $3(38)$ & 0.812 & 64 & 8 & $3(38)$ & 0.8 \\
\hline MS3 & 84 & 2 & $0(0)$ & 0.413 & 53 & 6 & $2(33)$ & 0.761 & 72 & 4 & $2(50)$ & 0.476 \\
\hline MS4 & 86 & 9 & $4(44)$ & 0.826 & 55 & 6 & $3(50)$ & 0.71 & 77 & 9 & $4(44)$ & 0.822 \\
\hline MS5 & 87 & 4 & $2(50)$ & 0.539 & 56 & 2 & $0(0)$ & 0.341 & 78 & 2 & $0(0)$ & 0.399 \\
\hline MS6 & 74 & 7 & $2(29)$ & 0.811 & 53 & 6 & $1(17)$ & 0.816 & 77 & 6 & $2(33)$ & 0.766 \\
\hline MS7 & 77 & 5 & $3(60)$ & 0.536 & 56 & 4 & $3(75)$ & 0.17 & 66 & 3 & $2(67)$ & 0.296 \\
\hline MS8 & 74 & 11 & $4(36)$ & 0.873 & 49 & 12 & $6(50)$ & 0.854 & 76 & 13 & $7(54)$ & 0.841 \\
\hline MS9 & 72 & 11 & $3(27)$ & 0.894 & 53 & 9 & $2(22)$ & 0.864 & 73 & 12 & $5(42)$ & 0.866 \\
\hline MS10 & 85 & 12 & $8(67)$ & 0.734 & 49 & 11 & $5(45)$ & 0.838 & 58 & 13 & $8(62)$ & 0.817 \\
\hline MS12 & 81 & 11 & 7 (64) & 0.779 & 52 & 13 & $4(31)$ & 0.912 & 76 & 10 & $4(40)$ & 0.838 \\
\hline MS15 & 84 & 8 & $3(38)$ & 0.81 & 55 & 7 & $3(43)$ & 0.789 & 75 & 7 & $3(43)$ & 0.739 \\
\hline MS16 & 70 & 18 & $9(50)$ & 0.902 & 48 & 15 & $7(47)$ & 0.895 & 62 & 15 & $6(40)$ & 0.901 \\
\hline MS20 & 82 & 8 & $2(25)$ & 0.844 & 55 & 12 & $3(25)$ & 0.905 & 68 & 13 & $4(31)$ & 0.9 \\
\hline Pv6635 & 84 & 12 & $4(33)$ & 0.891 & 54 & 9 & $4(44)$ & 0.82 & 73 & 9 & $4(44)$ & 0.814 \\
\hline
\end{tabular}

$N$ number of samples that amplified per locus, Na number of alleles, MAF number of alleles at minor allele frequency (frequency less than $5 \%$ ), He unbiased expected heterozygosity

primary molecular marker data must be able to differentiate alleles of close but non-identical size, such as those in the present study that differ in size by 3 bp or less. The QIAxcel Advanced System did not demonstrate sufficient reproducibility or accuracy (i.e., to measure the bp of a sequenced PCR product (range of \pm 3 base pairs)) to enable accurate data interpretation. These results were largely similar to previous (but smaller scale) studies [41]. The data provided here was based on comparison of a gold standard-a sequenced 227 bp PCR product-and using a sequencing gel measurement as a benchmark. This PCR product did not show any stutter or non-specific product, errors which are common in microsatellite analysis. Therefore, with microsatellite products with genotyping errors (i.e., stutters, additionally added adenines, non-specific peaks) the range of variation could be higher as the presence of stutters could affect the migration patterns of molecules on the capillary electrophoresis. In this sense, most of the degenerated microsatellite markers used in this study showed a great number of non-specific products, especially MS8 which showed four to six non-specific products near the principal allele peak.

Discrepancies between ABI PRISM and QIAxcel results were also seen. Twenty seven samples were genotyped by both platforms using five microsatellites markers. QIAxcel not only gave different product sizes than those observed on the ABI PRISM, but more, inaccurate alleles per locus were produced, which altered potential data interpretation (Additional file 3). Even though results were different, they were correlated between each other and those additional alleles observed were easily detected and edited to its correct allele size. However, when more replicates were done the variation of results increase, making more difficult to assign the correct value (Additional file 4). Differences between labelled and non-labelled products were seen too, but it was no as high as differences between QIAxcel and ABI platform (Additional files 5, 6). This heterogeneity of results was reflected in the analysis of the 271 samples. This analysis resulted in high diversity of markers and high number of alleles per locus. Though most markers were supposed to differ in three base pairs, differences in just one base pair were usually seen. The error produced by the QIAxcel Advanced System was reduced by transforming raw data to allele profile codes. Even though the expected $\mathrm{He}$ in each locality was lower and probably more accurate using the transformation (i.e., more lumping, less splitting), the genetic diversity of the three localities found in this study was somewhat higher than previously reported in the same region using the same markers but standard methods based on fluorophore-labelled primers [11, 25, 42].

Another limitation to the generalizability of the results presented here is the high rate of missing data that was found in 13 microsatellite markers. A particular case was MS16, which showed that $18 \%$ of data points were missing (72\% due to null alleles), higher than for the other microsatellites. This result possibly suggests that a mutation in the annealing region of one of these primers did not allow this product to amplify. Because of the high rate 
of missing data (more than $5 \%$ for 13 of the 15 microsatellites used) and the overestimation of diversity, more indepth analyses, such as pair-wise linkage disequilibrium and genetic differentiation between populations were not able to be performed since those could lead to erroneous interpretations.

Current malaria epidemiology research relies substantially on the use of molecular markers to estimate the malaria transmission dynamics. For this reason, genotyping methods with low cost of reagents, high accuracy and repeatability are needed. Recently analysis of single nucleotide polymorphisms (SNPs) have been shown to reliably to infer malaria transmission patterns in hyperendemic regions [43]. However, in low transmission regions where the parasite diversity is low associated with a clonal expansion of parasites, microsatellites are still the primary tool for detecting the small differences between parasite strains so that one can correctly infer transmission dynamics and potential outcomes of control measures [7, 10, 11, 29].

The automated QIAxcel advanced system is marketed as a rapid, facile and low-cost method for genotyping. Its main advantage is that it not depends on labelled primers with fluorophores which reduce the cost of the genotyping process drastically. While a 100 nano moles of a common primer is around 10 dollars, just 10 nano moles of a labelled primer is around 80 dollars. This difference makes the QIAxcel System an attractive option, but due to this characteristic, it is not possible to run the size marker and the sample into the same well and just one well contain the size marker in a plate, and the other wells contain the samples and the alignment markers. This work shows that each independent capillary and cartridge has different patterns of migration, and that the variation of results increases over the time. This heterogeneity is supposed to be reduced with the use of an alignment marker that normalizes the pattern of migration of molecules between capillaries, cartridges and runs. As the alignment marker flanks the PCR product in a range of 600 nucleotides the normalization process is not accurate enough to differentiate products that differ in $3 \mathrm{bp}$.

\section{Conclusion}

The use of affordable platforms that can generate data from fast evolving molecular markers, such as microsatellites, could facilitate scaling up molecular epidemiologic investigations in $P$. vivax and other eukaryotic pathogens. Several platforms and techniques have been used for this purpose; however, systematic assessment of the performance (precision, accuracy and reproducibility) of such systems is often lacking. This study found that one such system, the QIAxcel Advanced System, was not a reliable platform for $P$. vivax genotyping using microsatellites.

\section{Additional files}

Additional file 1: Microsatellites analyzed: chromosomal location, motif, and primers.

Additional file 2: A sequenced PCR product of 227 base pairs used.

Additional file 3: Comparison of allele sizes using QIAxcel vs $A B \mid$ Instruments.

Additional file 4: QIAxcel analysis of microsatellites 9 and 15 revealed too many alleles due to imprecision.

Additional file 5: Differences between labelled and unlabelled primers of Microsatellite 9 .

Additional file 6: Differences between labelled and unlabelled primers of Microsatellite 15.

\section{Authors' contributions}

PM carried out the PCR amplifications, participated in the electrophoresis analysis, performed the statistical analysis and drafted the manuscript. $\mathrm{MH}$ carried out the PCR amplifications, participated in the electrophoresis analysis and participated in the design of the study. MF carried out the PCR diagnosis of samples. ON participated in the design of the study and performed the statistical analysis. YP carried out the PCR diagnosis of samples. MC carried out the PCR diagnosis of samples. MNK participated in the design of the study. RHG participated in the design of the study. DG conceived of the study, and participated in its design and coordination. JV conceived of the study, and participated in its design. All authors read and approved the final manuscript.

\section{Author details}

${ }_{1}^{1}$ Malaria Laboratory, Institute of Tropical Medicine Alexander von Humboldt, Universidad Peruana Cayetano Heredia, Lima, Peru. ${ }^{2}$ Division of Infectious Diseases, Department of Medicine, University of California San Diego, La Jolla, CA, USA. ${ }^{3}$ Department of International Health, Johns Hopkins School of Public Health, Baltimore, MD, USA. ${ }^{4}$ Instituto Nacional de Salud, Lima, Peru. ${ }^{5}$ Departamento de Ciencias Celulares y Moleculares, Universidad Peruana Cayetano Heredia, Lima, Peru.

\section{Acknowledgements}

We wish to thank Paula Maguina of the University of California for her scientific, logistical and ethics compliance support that were essential for the completion of this work. This work was supported by US Public Health Service Grants U19AI089681, D43TW007120, R01AI067727, and K24Al068903 from the National Institutes of Health (JMV).

\section{Compliance with ethical guidelines}

\section{Competing interests}

The authors declare that they have no competing interests.

Received: 24 March 2015 Accepted: 8 August 2015

Published online: 21 August 2015

\section{References}

1. Arnott A, Barry AE, Reeder JC (2012) Understanding the population genetics of Plasmodium vivax is essential for malaria control and elimination. Malar J 11:14

2. Imwong M, Snounou G, Pukrittayakamee S, Tanomsing N, Kim JR, Nandy A et al (2007) Relapses of Plasmodium vivax infection usually result from activation of heterologous hypnozoites. J Infect Dis 195:927-933

3. Restrepo E, Imwong M, Rojas W, Carmona-Fonseca J, Maestre A (2011) High genetic polymorphism of relapsing P. vivax isolates in northwest Colombia. Acta Trop 119:23-29 
4. Imwong M, Boel ME, Pagornrat W, Pimanpanarak M, McGready R, Day NP et al (2012) The first Plasmodium vivax relapses of life are usually genetically homologous. J Infect Dis 205:680-683

5. Lin JT, Juliano JJ, Kharabora O, Sem R, Lin FC, Muth S et al (2012) Individual Plasmodium vivax msp1 variants within polyclonal $P$. vivax infections display different propensities for relapse. J Clin Microbiol 50:1449-1451

6. Abdullah NR, Barber BE, William T, Norahmad NA, Satsu UR, Muniandy PK, Ismail Z et al (2013) Plasmodium vivax population structure and transmission dynamics in Sabah Malaysia. PLoS One 8:e82553

7. Imwong M, Nair S, Pukrittayakamee S, Sudimack D, Williams JT, Mayxay $M$ et al (2007) Contrasting genetic structure in Plasmodium vivax populations from Asia and South America. Int J Parasitol 37:1013-1022

8. Raza A, Ghanchi NK, Thaver AM, Jafri S, Beg MA (2013) Genetic diversity of Plasmodium vivax clinical isolates from southern Pakistan using pvcsp and pvmsp1 genetic markers. Malar J 12:16

9. Zhong D, Bonizzoni M, Zhou G, Wang G, Chen B, Vardo-Zalik A et al (2011) Genetic diversity of Plasmodium vivax malaria in China and Myanmar. Infect Genet Evol 11:1419-1425

10. Chenet SM, Schneider KA, Villegas L, Escalante AA (2012) Local population structure of Plasmodium: impact on malaria control and elimination. Malar J 11:412

11. Delgado-Ratto C, Soto-Calle VE, Van den Eede P, Gamboa D, Rosas A Abatih EN et al (2014) Population structure and spatio-temporal transmission dynamics of Plasmodium vivax after radical cure treatment in a rural village of the Peruvian Amazon. Malar J 13:8

12. Ferreira MU, Karunaweera ND, da Silva-Nunes M, da Silva NS, Wirth DF, Hartl DL (2007) Population structure and transmission dynamics of Plasmodium vivax in rural Amazonia. J Infect Dis 195:1218-1226

13. Gunawardena S, Karunaweera ND, Ferreira MU, Phone-Kyaw M, Pollack RJ, Alifrangis M et al (2010) Geographic structure of Plasmodium vivax: microsatellite analysis of parasite populations from Sri Lanka, Myanmar, and Ethiopia. Am J Trop Med Hyg 82:235-242

14. Gauthier C, Tibayrenc M (2005) Population structure of malaria parasites: the driving epidemiological forces. Acta Trop 94:241-250

15. Taylor JE, Pacheco MA, Bacon DJ, Beg MA, Machado RL, Fairhurst RM et al (2013) The evolutionary history of Plasmodium vivax as inferred from mitochondrial genomes: parasite genetic diversity in the Americas. Mol Biol Evol 30:2050-2064

16. Yalcindag E, Elguero E, Arnathau C, Durand P, Akiana J, Anderson TJ et al (2012) Multiple independent introductions of Plasmodium falciparum in South America. Proc Natl Acad Sci USA 109:511-516

17. Zhou Z, Griffing SM, de Oliveira AM, McCollum AM, Quezada WM, Arrospide $\mathrm{N}$ et al (2008) Decline in sulfadoxine-pyrimethamine-resistant alleles after change in drug policy in the Amazon region of Peru. Antimicrob Agents Chemother 52:739-741

18. Mascorro CN, Zhao K, Khuntirat B, Sattabongkot J, Yan G, Escalante AA et al (2005) Molecular evolution and intragenic recombination of the merozoite surface protein MSP-3alpha from the malaria parasite Plasmodium vivax in Thailand. Parasitology 131:25-35

19. Rice BL, Acosta MM, Pacheco MA, Escalante AA (2013) Merozoite surface protein-3 alpha as a genetic marker for epidemiologic studies in Plasmodium vivax: a cautionary note. Malar J 12:288

20. Zakeri S, Barjesteh H, Djadid ND (2006) Merozoite surface protein-3alpha is a reliable marker for population genetic analysis of Plasmodium vivax. Malar J 5:53

21. Li XM, Guo CK, Li JH, Huang YM, Du JF, Fu WZ (2005) [A discussion on the CSP genotyping of Plasmodium vivax and malaria control in five southern provinces of China] (in Chinese). Zhongguo Ji Sheng Chong Xue Yu Ji Sheng Chong Bing Za Zhi 23:274-276

22. Schousboe ML, Rajakaruna RS, Amerasinghe PH, Konradsen F, Ord R, Pearce R et al (2011) Analysis of polymorphisms in the merozoite surface protein-3alpha gene and two microsatellite loci in Sri Lankan Plasmodium vivax: evidence of population substructure in Sri Lanka. Am J Trop Med Hyg 85:994-1001

23. Chenet SM, Branch OH, Escalante AA, Lucas CM, Bacon DJ (2008) Genetic diversity of vaccine candidate antigens in Plasmodium falciparum isolates from the Amazon basin of Peru. Malar J 7:93

24. Neafsey DE, Galinsky K, Jiang RH, Young L, Sykes SM, Saif S et al (2012) The malaria parasite Plasmodium vivax exhibits greater genetic diversity than Plasmodium falciparum. Nat Genet 44:1046-1050
25. Van den Eede P, Van der Auwera G, Delgado C, Huyse T, Soto-Calle VE, Gamboa D et al (2010) Multilocus genotyping reveals high heterogeneity and strong local population structure of the Plasmodium vivax population in the Peruvian Amazon. Malar J 9:151

26. Iwagami M, Hwang SY, Kim SH, Park SJ, Lee GY, Matsumoto-Takahashi et al (2013) Microsatellite DNA analysis revealed a drastic genetic change of Plasmodium vivax population in the Republic of Korea during 2002 and 2003. PLoS Negl Trop Dis 7:e2522

27. Gomez JC, MCNamara DT, Bockarie MJ, Baird JK, Carlton JM, Zimmerman PA (2003) Identification of a polymorphic Plasmodium vivax microsatellite marker. Am J Trop Med Hyg 69:377-379

28. Orjuela-Sanchez P, Brandi MC, Ferreira MU (2013) Microsatellite analysis of malaria parasites. Methods Mol Biol 1006:247-258

29. Sutton PL (2013) A call to arms: on refining Plasmodium vivax microsatellite marker panels for comparing global diversity. Malar J 12:447

30. Mallick PK, Sutton PL, Singh R, Singh OP, Dash AP, Singh AK et al (2013) Microsatellite analysis of chloroquine resistance associated alleles and neutral loci reveal genetic structure of Indian Plasmodium falciparum. Infect Genet Evol 19:164-175

31. McCollum AM, Mueller K, Villegas L, Udhayakumar V, Escalante AA (2007) Common origin and fixation of Plasmodium falciparum dhfr and dhps mutations associated with sulfadoxine-pyrimethamine resistance in a low-transmission area in South America. Antimicrob Agents Chemother 51:2085-2091

32. Vinayak S, Alam MT, Mixson-Hayden T, McCollum AM, Sem R, Shah NK et al (2010) Origin and evolution of sulfadoxine resistant Plasmodium falciparum. PLoS Pathog 6:e1000830

33. Dean DA, Wadl PA, Hadziabdic D, Wang X, Trigiano RN (2013) Analyzing microsatellites using the QIAxcel system. Methods Mol Biol 1006:223-243

34. Mangold KA, Manson RU, Koay ES, Stephens L, Regner M, Thomson RB Jr et al (2005) Real-time PCR for detection and identification of Plasmodium spp. J Clin Microbiol 43:2435-2440

35. Park HM (2005) Linear regression models for panel data using SAS. LIMDEP, and SPSS. Indiana University, Stata

36. Carroll RJ, Schneider H (1985) A note on Levene's tests for equality of variances. Stat Probab Lett 3:191-194

37. Karunaweera N, Ferreira M, Hartl D, Wirth D (2007) Fourteen polymorphic microsatellite DNA markers for the human malaria parasite Plasmodium vivax. Mol Ecol Notes 7:172-175

38. Van den Eede P, Erhart A, Van der Auwera G, Van Overmeir C, Thang ND, le Hung $X$ et al (2010) High complexity of Plasmodium vivax infections in symptomatic patients from a rural community in central Vietnam detected by microsatellite genotyping. Am J Trop Med Hyg 82:223-227

39. Peakall R, Smouse PE (2012) GenAlEx 6.5: genetic analysis in Excel. Population genetic software for teaching and research — an update. Bioinformatics 28:2537-2539

40. Nei M, Roychoudhury AK (1974) Sampling variances of heterozygosity and genetic distance. Genetics 76:379-390

41. Xiao M, Kong F, Jin P, Wang Q, Xiao K, Jeoffreys N et al (2012) Comparison of two capillary gel electrophoresis systems for Clostridium difficile ribotyping, using a panel of ribotype 027 isolates and whole-genome sequences as a reference standard. J Clin Microbiol 50:2755-2760

42. Van den Eede P, Soto-Calle VE, Delgado C, Gamboa D, Grande T, Rodriguez $\mathrm{H}$ et al (2011) Plasmodium vivax sub-patent infections after radical treatment are common in Peruvian patients: results of a 1-year prospective cohort study. PLoS One 6:e16257

43. Baniecki ML, Faust AL, Schaffner SF, Park DJ, Galinsky K, Daniels RF et al (2015) Development of a single nucleotide polymorphism barcode to genotype Plasmodium vivax infections. PLoS Negl Trop Dis 9:e0003539 